1 Hacettepe Journal of Mathematics and Statistics

$\bigcap$ Volume 47 (6) (2018), 1578-1594

\title{
An improved cosine similarity measure for intuitionistic fuzzy sets and their applications to decision-making process
}

\author{
Harish Garg*
}

\begin{abstract}
In the present study, improved cosine similarity measure for an intuitionistic fuzzy sets (IFSs) has been proposed by considering the interaction between the pairs of the membership degrees. Pairs of membership, non-membership are to be considered as vector representation during the formulation. The shortcomings of the existing measures have been highlighted and overcome by using the proposed measure. Also, in order to deal with the situation where the elements in a set are correlative, weighted cosine similarity measure has been defined. Finally, multi-criteria decision making (MCDM) method, based on the proposed similarity measure, has been presented under intuitionistic fuzzy environment. Numerical examples, one from the investment the money and others from the pattern recognition and medical diagnosis, have been taken to demonstrate the efficiency of the proposed approach and compared their results with the existing approaches results.
\end{abstract}

Keywords: Cosine similarity measures, intuitionistic fuzzy set, pattern recognition, medical diagnosis, multi criteria decision making.

Mathematics Subject Classification (2010): 03B52, 28E10, 90B50, 03E72

Received : 12.03.2017 Accepted : 20.07.2017 Doi : 10.15672/HJMS.2017.510

\footnotetext{
*Thapar University, Patiala to Thapar Institute of Engineering and Technology, Deemed University Patiala, India

Email : harishg58iitr@gmail.com, URL:http://sites.google.com/site/harishg58iitr/
} 


\section{Introduction}

In many decision-making problems, it is difficult for a decision maker to give his assessments towards the object in crisp values due to ambiguity and incomplete information. Instead, it has become popular that these assessments are presented by a fuzzy set or extensions of the fuzzy set. Fuzzy set (FS)[30], proposed by Zadeh, is a powerful tool to deal with vagueness and has received much attention. After it, some extensions such as intuitionistic fuzzy set (IFS) [1], interval-valued intuitionistic fuzzy set (IVIFS) [2] etc., have been proposed by the researchers. Under these environments, various researchers have investigated these theories in the process of decision making in the different fields [10, 16, 28, 13, 9]. For instance, Xu [27] presented weighted averaging aggregation operators for different intuitionistic fuzzy numbers (IFNs). Garg [8] presented a generalized intuitionistic fuzzy interactive geometric aggregation operator using Einstein norm operations. Garg [10] presented a new generalized score function for ranking the different IVIFSs. Garg [12] presented improved operational laws for aggregating the different preferences of the decision makers under the intuitionistic fuzzy environment.

However, in the field of information measure theory, the concept of correlation, similarity, distance, divergence are of key importance in a number of theoretical and applied statistical inferences like decision-making, machine learning etc. In that direction, Chen $[4,5]$ presented the similarity measure between the vague sets. Hung and Yang [21] gave the similarity measures between the two different IFSs based on Hausdorff distance. Garg et al. [13] presented an entropy-based method for solving the multicriteria decision-making problem under the fuzzy environment. Szmidt and Kacprzyk [25] presented the similarity measures between the IFSs. Gerstenkorn and Manko [17] and Bustince and Burillo [3], respectively introduced the concept of the correlation coefficient of IFS and interval-valued IFS. Garg [10] presented novel correlation coefficients under the Pythagorean fuzzy set environment. Singh and Garg [24] developed the distance measures between the type-2 intuitionistic fuzzy sets. Garg et al. [14] threw light on a generalized entropy measure of order $\alpha$ and degree $\beta$ under the IFS environment and applied it to solve the decision-making problems. Recently, Garg [11] presented the distance and similarity measures for the intuitionistic multiplicative preference relation and applied them to solve the decision-making problems from the field of pattern recognition and medical diagnosis. Apart from these, the several types of similarity measures have been proposed by the researchers $[4,5,20,7,6,23,15,31,21,29]$ for solving the decision making problems under the IFS environment. Out of these various measures, correlation coefficient and their corresponding cosine similarity measure (CSM) are one of the important measures for measuring the degree of similarity between the IFSs. But from these existing studies, it has been analyzed that they have some sort of deficiencies. For instance, under the intuitionistic fuzzy environment, when we take any two IFSs in which one set has zero membership degree and the other set have zero non-membership degree then their corresponding correlation coefficients, as well as the cosine similarity measure, becomes zero. Thus, these existing measures are independent on the other nonzero of the IFSs and hence it gives inconsistent results during the ranking procedure. Furthermore, it has been analyzed that the existing measures are unable to consider the degree of the interaction between the pairs of the membership into the analysis and hence their corresponding measures and their results do not give the correct information to the decision makers'. In other words, there is no interaction between the degree of membership functions.

In this regards, the present paper has resolved these issues by proposing an improved cosine similarity measure between the two IFSs. For this, an interaction between the pairs of membership functions has been considered in terms of their hesitation degree 
and hence their corresponding operations has been built. Based on it, improved cosine and weighted cosine similarity measures have been proposed under the IFS environment. The performance of the proposed measure with respect to the several existing similarity measures has been tested. Finally, based on the proposed similarity measures, we have developed a decision-making approach for ranking the alternatives under the intuitionistic fuzzy environment. At length, we provide multi-criteria decision-making (MCDM) problem from the fields of decision making to validate the effectiveness and applicability of the proposed decision method. Results are compared with the various existing measures and show the superiority of the approach.

To do so, the rest of the manuscript is organized as follows. Section 2 describes the basic definition of the IFS, correlation coefficient, cosine similarity measure along with their shortcomings. Section 3 presented improved operational laws between the two IFSs and hence based on it, an improved cosine and weighted cosine similarity measures have been proposed under the intuitionistic fuzzy environment. Section 4 describe the decisionmaking approach based on the proposed measure and hence validated with numerical examples of pattern recognition, medical diagnosis etc. A comparison analysis with some other existing measures has been investigated also in this section. A conclusion has been summarized in section 5 .

\section{Preliminaries}

In this section, some basic concepts on the IFSs and the cosine similarity measures have been defined over the universal set $X$.

2.1. Definition. An IFS $A$ in a finite universe of discourse $X=\left\{x_{1}, x_{2}, \ldots, x_{n}\right\}$ is defined as [1]

$$
A=\left\{\left\langle x, \mu_{A}(x), \nu_{A}(x)\right\rangle \mid x \in X\right\}
$$

where $\mu_{A}(x), \nu_{A}(x)$ represent the degrees of membership and non-membership of the element $x \in X$ to the set $A$, respectively, such that their sum is atmost one and $\pi_{A}(x)=$ $1-\mu_{A}(x)-\nu_{A}(x)$ is called the degree of hesitation of $x$ to $A$. For a given $x$, the pair $A=\langle\mu, \nu\rangle$ is called intuitionistic fuzzy value (IFV) or intuitionistic fuzzy number (IFN) where $\mu \in[0,1], \nu \in[0,1], \mu+\nu \leq 1$.

2.2. Definition. Let $A=\langle\mu, \nu\rangle, A_{1}=\left\langle\mu_{1}, \nu_{1}\right\rangle$ and $A_{2}=\left\langle\mu_{2}, \nu_{2}\right\rangle$ be three IFNs and for any real number $\lambda>0$, the basic operational rules between them are defined as follows [26].

(a) $A_{1} \oplus A_{2}=\left\langle 1-\left(1-\mu_{1}\right)\left(1-\mu_{2}\right), \nu_{1} \nu_{2}\right\rangle$

(b) $A_{1} \otimes A_{2}=\left\langle\mu_{1} \mu_{2}, 1-\left(1-\nu_{1}\right)\left(1-\nu_{2}\right)\right\rangle$

(c) $\lambda A=\left\langle 1-(1-\mu)^{\lambda}, \nu^{\lambda}\right\rangle$

(d) $A^{\lambda}=\left\langle\mu^{\lambda}, 1-(1-\nu)^{\lambda}\right\rangle$

2.3. Definition. [17] Let $X=\left\{x_{1}, x_{2}, \ldots, x_{n}\right\}$ be a finite fixed set, $A=\left\langle x_{i}, \mu_{A}\left(x_{i}\right), \nu_{A}\left(x_{i}\right)\right\rangle$ and $B=\left\langle x_{i}, \mu_{B}\left(x_{i}\right), \nu_{B}\left(x_{i}\right)\right\rangle$ be two IFSs. If

$$
k(A, B)=\frac{C(A, B)}{\sqrt{T(A) \cdot T(B)}}
$$

where $C(A, B)=\sum_{i=1}^{n}\left(\mu_{A}\left(x_{i}\right) \mu_{B}\left(x_{i}\right)+\nu_{A}\left(x_{i}\right) \nu_{B}\left(x_{i}\right)\right)$ be the covariance between $A$ and $B ; T(A)=\sum_{i=1}^{n}\left(\mu_{A}^{2}\left(x_{i}\right)+\nu_{A}^{2}\left(x_{i}\right)\right)$ and $T(B)=\sum_{i=1}^{n}\left(\mu_{B}^{2}\left(x_{i}\right)+\nu_{B}^{2}\left(x_{i}\right)\right)$ be the informational energies of the IMS $A$ and $B$ respectively, then $k(A, B)$ is called the correlation coefficient of the IFSs $A$ and $B$. 
2.4. Definition. A cosine similarity measure between two IFSs $A$ and $B$ is defined as [29]

$$
C_{I F S}(A, B)=\frac{1}{n} \sum_{i=1}^{n} \frac{\mu_{A}\left(x_{i}\right) \mu_{B}\left(x_{i}\right)+\nu_{A}\left(x_{i}\right) \nu_{B}\left(x_{i}\right)}{\sqrt{\mu_{A}^{2}\left(x_{i}\right)+\nu_{A}^{2}\left(x_{i}\right)} \sqrt{\mu_{B}^{2}\left(x_{i}\right)+\nu_{B}^{2}\left(x_{i}\right)}}
$$

where $0 \leq C_{I F S}(A, B) \leq 1$.

From Eq.(2.2), it has been observed that under some circumstances, this measure will not give some suitable decision to the decision makers to rank the different alternatives. These shortcomings have been explained with some counter examples as follows.

2.5. Example. Let $A_{1}, A_{2}$ and $A_{3}$ be three IFSs defined over $X=\left\{x_{1}, x_{2}\right\}$ given by $A_{1}=\left\{\left(x_{1}, 0.10,0.15\right),\left(x_{2}, 0.10,0.15\right)\right\}, A_{2}=\left\{\left(x_{1}, 0.20,0.30\right),\left(x_{2}, 0.20,0.30\right)\right\}$ and $A_{3}=\left\{\left(x_{1}, 0.30,0.45\right),\left(x_{2}, 0.30,0.45\right)\right\}$. Then, by utilizing Eq. $(2.2)$, we get $C_{I F S}\left(A_{1}, A_{2}\right)$ $=C_{I F S}\left(A_{2}, A_{3}\right)=C_{I F S}\left(A_{3}, A_{1}\right)$ and hence measure defined in Eq. (2.2) are unable to distinguish between the IFSs $A_{1}, A_{2}$ and $A_{3}$. Therefore, it is unable to give the correct order of the sets.

2.6. Example. Consider two IFNs $A=\langle 0.4,0\rangle$ and $B=\langle 0,0.5\rangle$, then by utilizing (2.2), we get $C_{I F S}(A, B)=0$ which means that there is no relationship between them. Moreover, if we take two IFNs $A=\langle\alpha, 0\rangle$ and $B=\langle 0, \beta\rangle$, where $0 \leq \alpha, \beta \leq 1$ then by Eq. (2.2), we get $C_{I F S}(A, B)=0$ which means that it is independent of the degrees of $\alpha$ and $\beta$ and therefore, it gives an undesirable result to the decision-makers.

2.7. Example. If we consider the two IFNs $A=\langle 0.4,0.3\rangle$ and $B=\langle 0,0.5\rangle$ then by utilizing (2.2), we get

$$
C_{I F S}(A, B)=\frac{(0.4)(0)+(0.3)(0.5)}{\sqrt{0.4^{2}+0.3^{2}} \sqrt{0^{2}+0.5^{2}}}=0.6
$$

Now, if we take two different IFNs $C=\langle 0.1,0.4898\rangle$ and $D=\langle 0.45189,0.21398\rangle$ respectively, then we get

$$
C_{I F S}(C, D)=\frac{(0.1)(0.45189)+(0.4898)(0.21398)}{\sqrt{0.1^{2}+0.4898^{2}} \sqrt{0.45189^{2}+0.21398^{2}}}=0.6
$$

Since $C_{I F S}(A, B)=C_{I F S}(C, D)$, but it has been clearly seen that IFNs $A$ and $B$ is different from $C$ and $D$. Hence, the results computed by the existing measure (2.2) is inconsistent.

Therefore, from these examples, we have concluded that the existing cosine similarity measure is unable to give the correct decision to the decision makers while ranking the numbers and hence there is a necessary to enhance these measures. In the next section, we have resolved this issue by defining a new measure based on some new operational laws and by taking the proper interaction between the pairs of the membership degrees.

\section{Proposed improved cosine similarity measure}

In this section, we have presented some improved form of the cosine similarity measures to compute the similarity index between the two IFSs defined over the fixed set $X$. Firstly, we have defined some improved operational laws between the pairs of the IFNs as follows.

3.1. Definition. Let $A=\left\langle\mu_{A}, \nu_{A}\right\rangle$ and $B=\left\langle\mu_{B}, \nu_{B}\right\rangle$ be two IFNs, $\lambda>0$ be a real number then operational rules between them are defined as follows $[18,19]$

(a) $A \oplus B=\left\langle 1-\left(1-\mu_{A}\right) \cdot\left(1-\mu_{B}\right),\left(1-\mu_{A}\right) \cdot\left(1-\mu_{B}\right)-\left(1-\mu_{A}-\nu_{A}\right) \cdot\left(1-\mu_{B}-\nu_{B}\right)\right\rangle$;

(b) $A \otimes B=\left\langle\left(1-\nu_{A}\right) \cdot\left(1-\nu_{B}\right)-\left(1-\mu_{A}-\nu_{A}\right) \cdot\left(1-\mu_{B}-\nu_{B}\right), 1-\left(1-\nu_{A}\right) \cdot\left(1-\nu_{B}\right)\right\rangle$; 
(c) $\lambda \cdot A=\left\langle 1-\left(1-\mu_{A}\right)^{\lambda},\left(1-\mu_{A}\right)^{\lambda}-\left(1-\mu_{A}-\nu_{A}\right)^{\lambda}\right\rangle$;

(d) $A^{\lambda}=\left\langle\left(1-\nu_{A}\right)^{\lambda}-\left(1-\mu_{A}-\nu_{A}\right)^{\lambda}, 1-\left(1-\nu_{A}\right)^{\lambda}\right\rangle$.

From these operational laws, it is clearly seen that the nonmembership part of the $A \oplus B$ contains the pairs $\mu_{A} \cdot \nu_{B}$ and $\mu_{B} \cdot \nu_{A}$ while membership function of $A \oplus B$ does not contain these pairs. For instance, the pair $\mu_{B} \cdot \nu_{A}$ represent the probability of $\mu_{B}$ and $\nu_{A}$ occurring simultaneously. Therefore, we can say that the influence on non-membership function is greater than that of membership function. It implies that attitude of the decision maker is optimistic. On the other hand for the pair of $A \otimes B$, these pairs have an effect on the membership functions while non-membership function of $A \otimes B$ does not contains. Thus in such circumstances, the attitude of the decision maker is pessimistic.

Based on these improved operational laws, we define the cosine similarity measures between two IFSs $A$ and $B$ as follows.

Consider $A=\left\{\left\langle x_{i}, \mu_{A}\left(x_{i}\right), \nu_{A}\left(x_{i}\right)\right\rangle \mid x_{i} \in X\right\}$ and $B=\left\{\left\langle x_{i}, \mu_{B}\left(x_{i}\right), \nu_{B}\left(x_{i}\right)\right\rangle \mid x_{i} \in X\right\}$ be two IFSs defined over the universal set $X$ then based on the improved operational laws between IFSs, the informational energies of the elements between them are defined as

$$
T_{A}\left(x_{i}\right)=\left(1-\nu_{A}\left(x_{i}\right)\right)^{2}+\left(1-\mu_{A}\left(x_{i}\right)\right)^{2}+2\left(1-\mu_{A}\left(x_{i}\right)-\nu_{A}\left(x_{i}\right)\right)^{2}
$$

and

$$
T_{B}\left(x_{i}\right)=\left(1-\nu_{B}\left(x_{i}\right)\right)^{2}+\left(1-\mu_{B}\left(x_{i}\right)\right)^{2}+2\left(1-\mu_{B}\left(x_{i}\right)-\nu_{B}\left(x_{i}\right)\right)^{2}
$$

Further, the correlation between two IFSs is defined as

$$
\begin{array}{r}
C_{(A, B)}\left(x_{i}\right)=\prod_{j=\{A, B\}}\left(1-\nu_{j}\left(x_{i}\right)\right)+\prod_{j=\{A, B\}}\left(1-\mu_{j}\left(x_{i}\right)\right) \\
+2 \prod_{j=\{A, B\}}\left(1-\mu_{j}\left(x_{i}\right)-\nu_{j}\left(x_{i}\right)\right)
\end{array}
$$

From the above, it is obvious that Eq. (3.3) satisfy the following properties for each $x_{i} \in X(i=1,2, \ldots, n)$ :

(P1) $C_{(A, A)}\left(x_{i}\right)=T_{A}\left(x_{i}\right)$

(P2) $C_{(A, B)}\left(x_{i}\right)=C_{(B, A)}\left(x_{i}\right)$

3.2. Definition. Let $A=\left\{\left\langle x_{i}, \mu_{A}\left(x_{i}\right), \nu_{A}\left(x_{i}\right)\right\rangle \mid x_{i} \in X\right\}$ and $B=\left\{\left\langle x_{i}, \mu_{B}\left(x_{i}\right), \nu_{B}\left(x_{i}\right)\right\rangle \mid\right.$ $\left.x_{i} \in X\right\}$, respectively be the sets of two IFSs defined on a universe of discourse $X$. Then, the improved cosine similarity measure $\left(I C_{I F S}\right)$ between them is defined as

$$
\begin{gathered}
I_{C_{I F S}(A, B)=\frac{1}{n} \sum_{i=1}^{n} \frac{C_{A, B}\left(x_{i}\right)}{\sqrt{T_{A}\left(x_{i}\right) \cdot T_{B}\left(x_{i}\right)}}}=\frac{1}{n} \sum_{i=1}^{n} \frac{\left\{\begin{array}{l}
\left(1-\nu_{A}\left(x_{i}\right)\right) \cdot\left(1-\nu_{B}\left(x_{i}\right)\right)+\left(1-\mu_{A}\left(x_{i}\right)\right) \cdot\left(1-\mu_{B}\left(x_{i}\right)\right) \\
+2\left(1-\mu_{A}\left(x_{i}\right)-\nu_{A}\left(x_{i}\right)\right) \cdot\left(1-\mu_{B}\left(x_{i}\right)-\nu_{B}\left(x_{i}\right)\right)
\end{array}\right\}}{\sqrt{\left\{\begin{array}{l}
\left.\left(1-\nu_{A}\left(x_{i}\right)\right)^{2}+\left(1-\mu_{A}\left(x_{i}\right)\right)^{2}+2\left(1-\mu_{A}\left(x_{i}\right)-\nu_{A}\left(x_{i}\right)\right)^{2}\right\} \\
\left\{\left(1-\nu_{B}\left(x_{i}\right)\right)^{2}+\left(1-\mu_{B}\left(x_{i}\right)\right)^{2}+2\left(1-\mu_{B}\left(x_{i}\right)-\nu_{B}\left(x_{i}\right)\right)^{2}\right\}
\end{array}\right.}}
\end{gathered}
$$

3.3. Theorem. The improved cosine similarity measure, as defined in Eq. (3.4), between the two IFSs $A$ and $B$ satisfy the following properties:

(P1) $I C_{I F S}(A, B)=I C_{I F S}(B, A)$

(P2) $0 \leq I C_{I F S}(A, B) \leq 1$ 
(P3) $\operatorname{IC}_{I F S}(A, B)=1$, if and only if $A=B$ i.e., $\mu_{A}\left(x_{i}\right)=\mu_{B}\left(x_{i}\right)$ and $\nu_{A}\left(x_{i}\right)=\nu_{B}\left(x_{i}\right)$ for $i=1,2, \ldots, n$

(P4) $I C_{I F S}(A, C) \leq I C_{I F S}(A, B)$ and $I C_{I F S}(A, C) \leq I C_{I F S}(B, C)$, if $A \subseteq B \subseteq C$ for an IFS $C$.

Proof. Consider two IFSs $A=\left\{\left\langle x_{i}, \mu_{A}\left(x_{i}\right), \nu_{A}\left(x_{i}\right)\right\rangle \mid x_{i} \in X\right\}$ and $B=\left\{\left\langle x_{i}, \mu_{B}\left(x_{i}\right), \nu_{B}\left(x_{i}\right)\right\rangle \mid\right.$ $\left.x_{i} \in X\right\}$ such that for each $x_{i} \in X, \mu_{A}, \nu_{A}, \mu_{B}, \nu_{B} \in[0,1]$ and $\mu_{A}+\nu_{A} \leq 1, \mu_{B}+\nu_{B} \leq 1$.

Then we have,

(P1) By the definition of $I C_{I F S}$, we get

$$
\begin{aligned}
& I C_{I F S}(A, B)=\frac{1}{n} \sum_{i=1}^{n} \frac{\left\{\begin{array}{l}
\left(1-\nu_{A}\left(x_{i}\right)\right) \cdot\left(1-\nu_{B}\left(x_{i}\right)\right)+\left(1-\mu_{A}\left(x_{i}\right)\right) \cdot\left(1-\mu_{B}\left(x_{i}\right)\right) \\
+2\left(1-\mu_{A}\left(x_{i}\right)-\nu_{A}\left(x_{i}\right)\right) \cdot\left(1-\mu_{B}\left(x_{i}\right)-\nu_{B}\left(x_{i}\right)\right)
\end{array}\right\}}{\left\{\left\{\left(1-\nu_{A}\left(x_{i}\right)\right)^{2}+\left(1-\mu_{A}\left(x_{i}\right)\right)^{2}+2\left(1-\mu_{A}\left(x_{i}\right)-\nu_{A}\left(x_{i}\right)\right)^{2}\right\}\right.} \\
& \sqrt{\left\{\left(1-\nu_{B}\left(x_{i}\right)\right)^{2}+\left(1-\mu_{B}\left(x_{i}\right)\right)^{2}+2\left(1-\mu_{B}\left(x_{i}\right)-\nu_{B}\left(x_{i}\right)\right)^{2}\right\}} \\
& =\frac{1}{n} \sum_{i=1}^{n} \frac{\left\{\begin{array}{l}
\left(1-\nu_{B}\left(x_{i}\right)\right) \cdot\left(1-\nu_{A}\left(x_{i}\right)\right)+\left(1-\mu_{B}\left(x_{i}\right)\right) \cdot\left(1-\mu_{A}\left(x_{i}\right)\right) \\
+2\left(1-\mu_{B}\left(x_{i}\right)-\nu_{B}\left(x_{i}\right)\right) \cdot\left(1-\mu_{A}\left(x_{i}\right)-\nu_{A}\left(x_{i}\right)\right)
\end{array}\right\}}{\left\{\left(1-\nu_{B}\left(x_{i}\right)\right)^{2}+\left(1-\mu_{B}\left(x_{i}\right)\right)^{2}+2\left(1-\mu_{B}\left(x_{i}\right)-\nu_{B}\left(x_{i}\right)\right)^{2}\right\}} \\
& \sqrt{\left\{\left(1-\nu_{A}\left(x_{i}\right)\right)^{2}+\left(1-\mu_{A}\left(x_{i}\right)\right)^{2}+2\left(1-\mu_{A}\left(x_{i}\right)-\nu_{A}\left(x_{i}\right)\right)^{2}\right\}} \\
& =I C_{I F S}(B, A)
\end{aligned}
$$

(P2) It is quite obvious that $I_{I F S}(A, B) \geq 0$. In order to show $I_{I F S}(A, B) \leq 1$ for two IFSs $A$ and $B$. We use the well-known Cauchy-Schwarz inequality:

$$
\sum_{i=1}^{n} a_{i} b_{i} \leq \sqrt{\left(\sum_{i=1}^{n} a_{i}^{2}\right) \cdot\left(\sum_{i=1}^{n} b_{i}^{2}\right)}
$$

with equality if and only if the two vectors $a=\left(a_{1}, a_{2}, \ldots, a_{n}\right)$ and $b=\left(b_{1}, b_{2}, \ldots, b_{n}\right)$ are linearly dependent.

In terms of membership and non-membership functions for two IFSs $A$ and $B$, Eq. (3.3) yields to

$$
\begin{aligned}
C_{(A, B)}\left(x_{i}\right)= & \prod_{j=\{A, B\}}\left(1-\mu_{j}\left(x_{i}\right)\right)+\prod_{j=\{A, B\}}\left(1-\nu_{j}\left(x_{i}\right)\right)+2 \prod_{j=\{A, B\}}\left(1-\mu_{j}\left(x_{i}\right)-\nu_{j}\left(x_{i}\right)\right) \\
= & \left(1-\mu_{A}\left(x_{i}\right)\right)\left(1-\mu_{B}\left(x_{i}\right)\right)+\left(1-\nu_{A}\left(x_{i}\right)\right)\left(1-\nu_{B}\left(x_{i}\right)\right) \\
& +2\left(1-\mu_{A}\left(x_{i}\right)-\nu_{A}\left(x_{i}\right)\right)\left(1-\mu_{B}\left(x_{i}\right)-\nu_{B}\left(x_{i}\right)\right) \\
\leq & \sqrt{\left\{\left(1-\mu_{A}\left(x_{i}\right)\right)^{2}+\left(1-\nu_{A}\left(x_{i}\right)\right)^{2}+2\left(1-\mu_{A}\left(x_{i}\right)-\nu_{A}\left(x_{i}\right)\right)^{2}\right\}} \\
\leq & \sqrt{T(A) \cdot T(B)}
\end{aligned}
$$

Thus, from Eq. (3.4) we get, $I C_{I F S}(A, B) \leq 1$. Therefore, $0 \leq I C_{I F S}(A, B) \leq 1$. 
(P3) If $A=B$ i.e., $\mu_{A}\left(x_{i}\right)=\nu_{B}\left(x_{i}\right)$ and $\nu_{A}\left(x_{i}\right)=\nu_{B}\left(x_{i}\right)$ for all $x_{i} \in X$ then by Eq. (3.4), we get

$$
\begin{aligned}
I C_{I F S}(A, B)= & \frac{1}{n} \sum_{i=1}^{n} \frac{\left\{\begin{array}{l}
\left(1-\nu_{A}\left(x_{i}\right)\right) \cdot\left(1-\nu_{A}\left(x_{i}\right)\right)+\left(1-\mu_{A}\left(x_{i}\right)\right) \cdot\left(1-\mu_{A}\left(x_{i}\right)\right) \\
+2\left(1-\mu_{A}\left(x_{i}\right)-\nu_{A}\left(x_{i}\right)\right) \cdot\left(1-\mu_{A}\left(x_{i}\right)-\nu_{A}\left(x_{i}\right)\right)
\end{array}\right\}}{\left\{\begin{array}{l}
\left\{\left(1-\nu_{A}\left(x_{i}\right)\right)^{2}+\left(1-\mu_{A}\left(x_{i}\right)\right)^{2}+2\left(1-\mu_{A}\left(x_{i}\right)-\nu_{A}\left(x_{i}\right)\right)^{2}\right\} \\
\left\{\left(1-\nu_{A}\left(x_{i}\right)\right)^{2}+\left(1-\mu_{A}\left(x_{i}\right)\right)^{2}+2\left(1-\mu_{A}\left(x_{i}\right)-\nu_{A}\left(x_{i}\right)\right)^{2}\right\}
\end{array}\right.} \\
= & \frac{1}{n} \sum_{i=1}^{n} \frac{\left\{\left(1-\nu_{A}\left(x_{i}\right)\right)^{2}+\left(1-\mu_{A}\left(x_{i}\right)\right)^{2}+2\left(1-\mu_{A}\left(x_{i}\right)-\nu_{A}\left(x_{i}\right)\right)^{2}\right\}}{\left\{\left(1-\nu_{A}\left(x_{i}\right)\right)^{2}+\left(1-\mu_{A}\left(x_{i}\right)\right)^{2}+2\left(1-\mu_{A}\left(x_{i}\right)-\nu_{A}\left(x_{i}\right)\right)^{2}\right\}} \\
= & 1
\end{aligned}
$$

On the other hand, if we assume $\operatorname{IC}_{I F S}(A, B)=1$, then by Eq. (3.4), we conclude that

$$
\frac{\left\{\begin{array}{l}
\left(1-\nu_{A}\left(x_{i}\right)\right) \cdot\left(1-\nu_{B}\left(x_{i}\right)\right)+\left(1-\mu_{A}\left(x_{i}\right)\right) \cdot\left(1-\mu_{B}\left(x_{i}\right)\right) \\
+2\left(1-\mu_{A}\left(x_{i}\right)-\nu_{A}\left(x_{i}\right)\right) \cdot\left(1-\mu_{B}\left(x_{i}\right)-\nu_{B}\left(x_{i}\right)\right)
\end{array}\right\}}{\sqrt{\left\{\left(1-\nu_{A}\left(x_{i}\right)\right)^{2}+\left(1-\mu_{A}\left(x_{i}\right)\right)^{2}+2\left(1-\mu_{A}\left(x_{i}\right)-\nu_{A}\left(x_{i}\right)\right)^{2}\right\}} \cdot}=1
$$

Take $1-\nu_{A}\left(x_{i}\right)=a_{1}, 1-\nu_{B}\left(x_{i}\right)=a_{2}, 1-\mu_{A}\left(x_{i}\right)=b_{1}, 1-\mu_{B}\left(x_{i}\right)=b_{2}$, $1-\mu_{A}\left(x_{i}\right)-\nu_{A}\left(x_{i}\right)=c_{1}$ and $1-\mu_{B}\left(x_{i}\right)-\nu_{B}\left(x_{i}\right)=c_{2}$. Then, from (3.6), we get

$$
\begin{aligned}
& \frac{a_{1} a_{2}+b_{1} b_{2}+2 c_{1} c_{2}}{\sqrt{\left(a_{1}^{2}+b_{1}^{2}+2 c_{1}^{2}\right) \cdot\left(a_{2}^{2}+b_{2}^{2}+2 c_{2}^{2}\right)}}=1 \\
\Rightarrow \quad & \left(a_{1} a_{2}+b_{1} b_{2}+2 c_{1} c_{2}\right)^{2}=\left(a_{1}^{2}+b_{1}^{2}+2 c_{1}^{2}\right) \cdot\left(a_{2}^{2}+b_{2}^{2}+2 c_{2}^{2}\right) \\
\Rightarrow \quad & \left(a_{1} b_{2}-b_{1} a_{2}\right)^{2}+2\left(b_{1} c_{2}-b_{2} c_{1}\right)^{2}+2\left(a_{1} c_{2}-a_{2} c_{1}\right)^{2}=0
\end{aligned}
$$

which implies that $a_{1} b_{2}=b_{1} a_{2}, b_{1} c_{2}=b_{2} c_{1}$ and $a_{1} c_{2}=c_{1} a_{2}$. Thus, from it, we conclude that $\frac{a_{1}}{a_{2}}=\frac{b_{1}}{b_{2}}=\frac{c_{1}}{c_{2}}$ which implies that $a_{1}=h a_{2}, b_{1}=h b_{2}$ and $c_{1}=h c_{2}$ for some nonzero $h$. Therefore, $a_{1}+b_{1}-c_{1}=h\left(a_{2}+b_{2}-c_{2}\right)$ and by substituting the values of $a_{1}, b_{1}, c_{1}, a_{2}, b_{2}$ and $c_{2}$, we get $h=1$. Hence, $a_{1}=a_{2}, b_{1}=b_{2}$ and $c_{1}=c_{2}$ which further implies that $\nu_{A}\left(x_{i}\right)=\nu_{B}\left(x_{i}\right), \mu_{A}\left(x_{i}\right)=\mu_{B}\left(x_{i}\right)$ and get $A=B$.

Therefore, $I_{I F S}(A, B)=1$ if and only if $A=B$.

(P4) If $A \subseteq B \subseteq C$, geometrically the angle between $A$ and $C$ should be larger than the angle between $A$ and $B$ and the angle between $B$ and $C$ for any element $x_{i}$. Thus, the relation $I C_{I F S}(A, C) \leq I C_{I F S}(A, B)$ and $I C_{I F S}(A, C) \leq I C_{I F S}(B, C)$ can be obtained from Eq. (3.4).

Hence, $I C_{I F S}$ is a valid cosine similarity measure.

In order to show the superiority of the proposed measure defined in Eq. (3.4) against the existing one, the proposed measure has been tested on the above considered examples as given in section 2 where the existing measure fails to rank the different IFSs.

3.4. Example. If we utilize Eq. (3.4) to the considered data as given in Example 2.5 then their corresponding measurement values are computed as $\operatorname{IC}_{I F S}\left(A_{1}, A_{2}\right)=$ 
$0.9922 ; \operatorname{IC}_{I F S}\left(A_{2}, A_{3}\right)=0.9772$ and $\operatorname{IC}_{I F S}\left(A_{3}, A_{1}\right)=0.9430$. Thus, we conclude that the proposed measure has successfully overcome the shortcoming of the existing similarity measure, where Ye [29] gets incorrect results: $C_{I F S}\left(A_{1}, A_{2}\right)=C_{I F S}\left(A_{2}, A_{3}\right)=$ $C_{I F S}\left(A_{3}, A_{1}\right)$ as shown in Example 2.5.

3.5. Example. If we apply the proposed $I C_{I F S}$ on the data given in Example 2.6 then the corresponding value is $\operatorname{IC}_{I F S}(A, B)=0.8910$ which is non-zero. Thus, the proposed measure are capable to handle the problem when at least one degree of their corresponding IFN become zero.

3.6. Example. If we utilize proposed $I C_{I F S}$ on the data given in Example 2.7 i.e., for $A=\langle 0.4,0.3\rangle$ and $B=\langle 0,0.5\rangle$ then we get

$$
I_{I F S}(A, B)=\frac{(0.7)(0.5)+(0.6)(1)+2(0.3)(0.5)}{\sqrt{(0.6)^{2}+(0.7)^{2}+2(0.3)^{2}} \sqrt{(1)^{2}+(0.5)^{2}+2(0.5)^{2}}}=0.9310
$$

On the other hand, if we compute the $I C_{I F S}$ for the IFNs $C$ and $D$ then we get $I C_{I F S}(C, D)=0.9220$. Thus, it has been clearly seen that by changing the degree of the membership functions, their corresponding measure changes and hence their is an effect of the pair of the membership functions on proposed similarity measure.

Hence, the proposed measure has successfully overcome the shortcoming of the existing cosine similarity measure.

3.7. Example. Let $A=\left\{\left(x_{1}, 0.7,0.2\right),\left(x_{2}, 0.4,0.5\right),\left(x_{3}, 0.5,0.3\right)\right\}$ and $B=\left\{\left(x_{1}, 0.6\right.\right.$, $\left.0.4),\left(x_{2}, 0.3,0.6\right),\left(x_{3}, 0.7,0.2\right)\right\}$ be two IFSs defined over the finite universe $X=\left\{x_{1}, x_{2}, x_{3}\right\}$ then by using the Eq. (3.1), the informational energy of $A$ is

$$
\begin{aligned}
T_{A}\left(x_{i}\right)= & \left\{\left(1-\nu_{A}\left(x_{i}\right)\right)^{2}+\left(1-\mu_{A}\left(x_{i}\right)\right)^{2}+2\left(1-\mu_{A}\left(x_{i}\right)-\nu_{A}\left(x_{i}\right)\right)^{2}\right\} \\
= & \left\{0.8^{2}+0.3^{2}+2(1-0.7-0.2)^{2}, 0.5^{2}+0.6^{2}+2(1-0.4-0.5)^{2}\right. \\
& \left.0.7^{2}+0.5^{2}+2(1-0.5-0.3)^{2}\right\} \\
= & \{0.75,0.63,0.82\}
\end{aligned}
$$

and by Eq. (3.2), the informational energy of $B$ is

$$
\begin{aligned}
T_{B}\left(x_{i}\right)= & \left\{\left(1-\nu_{A}\left(x_{i}\right)\right)^{2}+\left(1-\mu_{A}\left(x_{i}\right)\right)^{2}+2\left(1-\mu_{A}\left(x_{i}\right)-\nu_{A}\left(x_{i}\right)\right)^{2}\right\} \\
= & \left\{0.6^{2}+0.4^{2}+2(1-0.6-0.4)^{2}, 0.4^{2}+0.7^{2}+2(1-0.3-0.6)^{2}\right. \\
& \left.0.8^{2}+0.3^{2}+2(1-0.7-0.2)^{2}\right\} \\
= & \{0.52,0.67,0.75\}
\end{aligned}
$$


On the other hand, by using the Eq. (3.3), the correlation between the intuitionistic fuzzy set $A$ and $B$ is

$$
\begin{aligned}
C_{(A, B)}\left(x_{i}\right)= & \left\{\prod_{j=A, B}\left(1-\nu_{j}\left(x_{i}\right)\right)+\prod_{j=A, B}\left(1-\mu_{j}\left(x_{i}\right)\right)+2 \prod_{j=A, B}\left(1-\mu_{j}\left(x_{i}\right)-\nu_{j}\left(x_{i}\right)\right)\right\} \\
= & \{(0.8)(0.6)+(0.3)(0.4)+2(0.1)(0),(0.5)(0.4)+(0.6)(0.7)+2(0.1)(0.1), \\
& (0.8)(0.7)+(0.5)(0.3)+2(0.2)(0.1)\} \\
= & \{0.60,0.64,0.75\}
\end{aligned}
$$

Hence, the cosine similarity measure between IFSs $A$ and $B$ is given by

$$
\begin{aligned}
I C_{I F S}(A, B) & =\frac{1}{3} \sum_{i=1}^{3} \frac{C_{(A, B)}\left(x_{i}\right)}{\sqrt{T_{A}\left(x_{i}\right) \cdot T_{B}\left(x_{i}\right)}} \\
& =\frac{1}{3}\left[\frac{0.60}{\sqrt{0.75 \times 0.52}}+\frac{0.64}{\sqrt{0.63 \times 0.67}}+\frac{0.75}{\sqrt{0.82 \times 0.75}}\right] \\
& =0.9674
\end{aligned}
$$

3.8. Remark. From Eq. (3.4), it has been observed the following points

(i) If $\mu\left(x_{i}\right)+\nu\left(x_{i}\right)=1$ for all $x_{i} \in X$ then the proposed $I C_{I F S}$ will be equivalent to the existing $C_{I F S}$.

(ii) If $n=1$ and $\mu\left(x_{i}\right)+\nu\left(x_{i}\right)=1$ for all $x_{i} \in X$ then the $\operatorname{IC}_{I F S}(A, B)$ becomes the correlation coefficient between two IFSs $A$ and $B$.

However, in many practical situations, the different set may have taken different weights and thus, weight $\omega_{i}$ of the element $x_{i} \in X(i=1,2, \ldots, n)$ should be taken into account. In the following, we develop weighted cosine similarity measures between the two IFSs. For it, let $\omega=\left(\omega_{1}, \omega_{2}, \ldots, \omega_{n}\right)^{T}$ be the weight vector of $x_{i}(i=1,2, \ldots, n)$ with $\omega_{i}>0$ and $\sum_{i=1}^{n} \omega_{i}=1$, then we have extended the above formulated cosine similarity coefficients $I C_{I F S}$ to weighted cosine similarity measures $W I C_{I F S}$ between two IFSs $A$ and $B$, as follows:

$$
\begin{gathered}
W C_{I F S}(A, B)=\sum_{i=1}^{n} \omega_{i} \frac{C_{A, B}\left(x_{i}\right)}{\sqrt{T_{A}\left(x_{i}\right) \cdot T_{B}\left(x_{i}\right)}} \\
\sum_{i=1}^{n} \omega_{i} \frac{\left\{\begin{array}{l}
\left.\left(1-\nu_{A}\left(x_{i}\right)\right) \cdot\left(1-\nu_{B}\left(x_{i}\right)\right)+\left(1-\mu_{A}\left(x_{i}\right)\right) \cdot\left(1-\mu_{B}\left(x_{i}\right)\right)\right\} \\
+2\left(1-\mu_{A}\left(x_{i}\right)-\nu_{A}\left(x_{i}\right)\right) \cdot\left(1-\mu_{B}\left(x_{i}\right)-\nu_{B}\left(x_{i}\right)\right)
\end{array}\right\}}{\sqrt{\left\{\left(1-\nu_{A}\left(x_{i}\right)\right)^{2}+\left(1-\mu_{A}\left(x_{i}\right)\right)^{2}+2\left(1-\mu_{A}\left(x_{i}\right)-\nu_{A}\left(x_{i}\right)\right)^{2}\right\}}} \\
\sqrt{\left.\left\{1-\nu_{B}\left(x_{i}\right)\right)^{2}+\left(1-\mu_{B}\left(x_{i}\right)\right)^{2}+2\left(1-\mu_{B}\left(x_{i}\right)-\nu_{B}\left(x_{i}\right)\right)^{2}\right\}}
\end{gathered}
$$

From the above it has been seen that if $\omega=(1 / n, 1 / n, \ldots, 1 / n)^{T}$, then Eq. (3.7) reduces to Eq. (3.4).

3.9. Theorem. Let $\omega=\left(\omega_{1}, \omega_{2}, \ldots, \omega_{n}\right)^{T}$ be the weight vector of $x_{i},(i=1,2, \ldots, n)$ with $\omega_{i}>0$ and $\sum_{i=1}^{n} \omega_{i}=1$, then the measure defined in Eq. (3.7), satisfy the following properties: 
(P1) $W I C_{I F S}(A, B)=W I C_{I F S}(B, A)$

(P2) $0 \leq W I C_{I F S}(A, B) \leq 1$

(P3) $W C_{I F S}(A, B)=1$, if and only if $A=B$ i.e. $\mu_{A}\left(x_{i}\right)=\mu_{B}\left(x_{i}\right)$ and $\nu_{A}\left(x_{i}\right)=$ $\nu_{B}\left(x_{i}\right)$ for $i=1,2, \ldots, n$

(P4) $W I_{I F S}(A, C) \leq W I C_{I F S}(A, B)$ and $W I C_{I F S}(A, C) \leq W I C_{I F S}(B, C)$, if $A \subseteq$ $B \subseteq C$ for an IFS $C$.

Proof. Proof follows from the above theorem, so we omit here.

Furthermore, various authors $[4,5,20,7,6,23,31,21,29]$ have proposed the different types of similarity measure under the IFSs environment, whose description are listed as below.

(i) $S_{C}(A, B)=1-\frac{\sum_{i=1}^{n}\left|\mu_{A}\left(x_{i}\right)-\nu_{A}\left(x_{i}\right)-\mu_{B}\left(x_{i}\right)+\nu_{B}\left(x_{i}\right)\right|}{2 n}$;

(ii) $S_{H}(A, B)=1-\frac{\sum_{i=1}^{n}\left(\left|\mu_{A}\left(x_{i}\right)-\mu_{B}\left(x_{i}\right)\right|\right)+\left(\left|\nu_{A}\left(x_{i}\right)-\nu_{B}\left(x_{i}\right)\right|\right)}{2 n}$.

(iii) $S_{L}(A, B)=1-\frac{\sum_{i=1}^{n}\left|S_{A}\left(x_{i}\right)-S_{B}\left(x_{i}\right)\right|}{4 n}-\frac{\sum_{i=1}^{n}\left|\mu_{A}\left(x_{i}\right)-\mu_{B}\left(x_{i}\right)\right|+\left|\nu_{A}\left(x_{i}\right)-\nu_{B}\left(x_{i}\right)\right|}{4 n}$

(iv) $S_{O}(A, B)=1-\sqrt{\frac{\sum_{i=1}^{n}\left(\mu_{A}\left(x_{i}\right)-\mu_{B}\left(x_{i}\right)\right)^{2}+\left(\nu_{A}\left(x_{i}\right)-\nu_{B}\left(x_{i}\right)\right)^{2}}{2 n}}$.

(v) $S_{D C}(A, B)=1-\sqrt[p]{\frac{\sum_{i=1}^{n}\left|\frac{\mu_{A}\left(x_{i}\right)-\nu_{A}\left(x_{i}\right)+\mu_{B}\left(x_{i}\right)-\nu_{B}\left(x_{i}\right)}{2}\right|^{p}}{n}}$,

(vi) $S_{H B}(A, B)=\frac{S_{D C}\left(\mu_{A}\left(x_{i}\right), \mu_{B}\left(x_{i}\right)\right)+S_{D C}\left(1-\nu_{A}\left(x_{i}\right), 1-\nu_{B}\left(x_{i}\right)\right)}{2}$

(vii) $S_{e}^{p}(A, B)=1-\sqrt[p]{\frac{\sum_{i=1}^{n}\left(\phi_{\mu}\left(x_{i}\right)+\phi_{\nu}\left(x_{i}\right)\right)^{p}}{n}}$, where $\phi_{\mu}(A, B)=\frac{\left|\mu_{A}\left(x_{i}\right)-\mu_{B}\left(x_{i}\right)\right|}{2} ; \phi_{\nu}\left(x_{i}\right)=$ $\frac{\left|\nu_{B}\left(x_{i}\right)-\nu_{A}\left(x_{i}\right)\right|}{2}$

(viii) $S_{s}^{p}(A, B)=1-\sqrt[p]{\frac{\sum_{i=1}^{n}\left(\phi_{s 1}\left(x_{i}\right)+\phi_{s 2}\left(x_{i}\right)\right)^{p}}{n}}$ where $\phi_{s 1}\left(x_{i}\right)=\frac{\left|m_{A 1}\left(x_{i}\right)-m_{B 1}\left(x_{i}\right)\right|}{2}$ and $\phi_{s 2}\left(x_{i}\right)=\frac{\left|m_{A 2}\left(x_{i}\right)-m_{B 2}\left(x_{i}\right)\right|}{2} ; m_{A 1}\left(x_{i}\right)=\frac{\mu_{A}\left(x_{i}\right)+m_{A}\left(x_{i}\right)}{2} ; m_{B 1}\left(x_{i}\right)=\frac{\mu_{B}\left(x_{i}\right)+m_{B}\left(x_{i}\right)}{2} ;$ $m_{A 2}\left(x_{i}\right)=\frac{m_{A}\left(x_{i}\right)+1-\nu_{A}\left(x_{i}\right)}{2} ; m_{B 2}\left(x_{i}\right)=\frac{m_{B}\left(x_{i}\right)+1-\nu_{B}\left(x_{i}\right)}{2} ; m_{A}\left(x_{i}\right)=\frac{\mu_{A}\left(x_{i}\right)+1-\nu_{A}\left(x_{i}\right)}{2}$ and $m_{B}\left(x_{i}\right)=\frac{\mu_{B}\left(x_{i}\right)+1-\nu_{B}\left(x_{i}\right)}{2}$

(ix) $S_{H Y}^{1}=1-d_{H}(A, B)$ where $d_{H}(A, B)=\frac{1}{n} \sum_{i=1}^{n} \max \left(\left|\mu_{A}\left(x_{i}\right)-\mu_{B}\left(x_{i}\right)\right|, \mid \nu_{A}\left(x_{i}\right)-\right.$ $\left.\nu_{B}\left(x_{i}\right) \mid\right)$

(x) $S_{H Y}^{2}=\left(e^{-d_{H}(A, B)}-e^{-1}\right) /\left(1-e^{-1}\right)$ for the same $d_{H}(A, B)$

(xi) $S_{H Y}^{3}=\left(1-d_{H}(A, B)\right) /\left(1+d_{H}(A, B)\right)$ for the same $d_{H}(A, B)$

Now, in order to compare the performance of the proposed measure with these existing measures, six different sets of IFSs $A$ and $B$ have been taken for an illustration which are mentioned in Table 1 . The results corresponding to the proposed measures along with the existing measures are given in Table 1. 
Table 1. Similarity measure between IFSs $A$ and $B$

\begin{tabular}{c|cccccc}
\hline & 1 & 2 & 3 & 4 & 5 & 6 \\
\hline$A$ & $(0.3,0.3)$ & $(0.3,0.4)$ & $(1,0)$ & $(0.5,0.5)$ & $(0.4,0.2)$ & $(0.4,0.2)$ \\
$B$ & $(0.4,0.4)$ & $(0.4,0.3)$ & $(0,0)$ & $(0,0)$ & $(0.5,0.3)$ & $(0.5,0.2)$ \\
\hline$S_{C}[4,5]$ & 1 & 0.9 & 0.5 & 1 & 1 & 0.95 \\
$S_{H}[20]$ & 0.9 & 0.9 & 0.5 & 0.5 & 0.9 & 0.95 \\
$S_{L}[7]$ & 0.95 & 0.9 & 0.5 & 0.75 & 0.95 & 0.95 \\
$S_{O}[7]$ & 0.9 & 0.9 & 0.3 & 0.5 & 0.9 & 0.93 \\
$S_{D C}[6]$ & 1 & 0.9 & 0.5 & 1 & 1 & 0.95 \\
$S_{H B}[23]$ & 0.9 & 0.9 & 0.5 & 0.5 & 0.9 & 0.95 \\
$S_{e}^{p}[31]$ & 0.9 & 0.9 & 0.5 & 0.5 & 0.9 & 0.95 \\
$S_{S}^{p}[31]$ & 0.95 & 0.9 & 0.5 & 0.75 & 0.95 & 0.95 \\
$S_{H Y}^{1}[21]$ & 0.9 & 0.9 & 0 & 0.5 & 0.9 & 0.9 \\
$S_{H Y}^{2}[21]$ & 0.85 & 0.85 & 0 & 0.38 & 0.85 & 0.85 \\
$S_{H Y}^{3}[21]$ & 0.82 & 0.82 & 0 & 0.33 & 0.82 & 0.82 \\
$C_{I F S}[29]$ & 1 & 0.96 & 0 & 0 & 0.9971 & 0.9965 \\
$I C_{I F S}$ (proposed) & 0.9806 & 0.9903 & 0.5 & 0.7071 & 0.9804 & 0.9929 \\
\hline
\end{tabular}

\section{A new method for intuitionistic fuzzy group decision making based on the proposed cosine similarity measures}

In this section, we have presented a decision-making approach based on the proposed cosine similarity measures under the intuitionistic fuzzy environment following by the three numerical examples.

4.1. Proposed decision making method. The following assumptions or notations are used to present the MCDM problems for evaluating of these with an intuitionistic fuzzy environment. Let $A=\left\{A_{1}, A_{2}, \ldots, A_{m}\right\}$ be the set of $m$ different alternatives which have to be evaluated under the set of $n$ different criteria $C=\left\{C_{1}, C_{2}, \ldots, C_{n}\right\}$. The weight vector information corresponding to each criterion is denoted by $\omega=\left(\omega_{1}, \omega_{2}, \ldots, \omega_{n}\right)^{T}$ with $w_{j}>0$ and $\sum_{j=1}^{n} \omega_{j}=1$. Assume that these alternatives $A_{i}(i=1,2, \ldots, m)$ are evaluated by an expert who will receive the full responsibility for the whole process and gives their preferences under the intuitionistic fuzzy environment. These preference values are summarized in the form of the decision matrix $D=\left(\alpha_{i j}\right)_{m \times n}$ where $\alpha_{i j}=\left\langle\mu_{i j}, \nu_{i j}\right\rangle$ represents the priority values of alternative $A_{i}$ given by decision maker such that $\mu_{i j}, \nu_{i j} \in[0,1]$ and $\mu_{i j}+\nu_{i j} \leq 1$. Then, the proposed method has been summarized into the various steps which are described as follows.

(Step 1:) Construct the intuitionistic fuzzy decision matrix $D=\left(\alpha_{i j}\right)_{m \times n}$ as.

$$
D=\begin{array}{cccc}
C_{1} & C_{2} & \ldots & C_{n} \\
A_{1} & A_{2} \\
\vdots & A_{m}
\end{array}\left(\begin{array}{cccc}
\left\langle\mu_{11}, \nu_{11}\right\rangle & \left\langle\mu_{12}, \nu_{12}\right\rangle & \ldots & \left\langle\mu_{1 n}, \nu_{1 n}\right\rangle \\
\left\langle\mu_{21}, \nu_{21}\right\rangle & \left\langle\mu_{22}, \nu_{22}\right\rangle & \ldots & \left\langle\mu_{2 n}, \nu_{2 n}\right\rangle \\
\vdots & \vdots & \ddots & \vdots \\
\left\langle\mu_{m 1}, \nu_{m 1}\right\rangle & \left\langle\mu_{m 2}, \nu_{m 2}\right\rangle & \ldots & \left\langle\mu_{m n}, \nu_{m n}\right\rangle
\end{array}\right)
$$


(Step 2:) Utilize appropriately the $I C_{I F S}$ measure:

$$
\begin{aligned}
& I C_{I F S}\left(A_{i}, B\right)=\frac{1}{n} \sum_{j=1}^{n} \frac{\left\{\begin{array}{l}
\left(1-\nu_{A_{i}}\left(C_{i j}\right)\right) \cdot\left(1-\nu_{B}\left(C_{i j}\right)\right)+\left(1-\mu_{A_{i}}\left(C_{i j}\right)\right) \cdot\left(1-\mu_{B}\left(C_{i j}\right)\right) \\
+2\left(1-\mu_{A_{i}}\left(C_{i j}\right)-\nu_{A_{i}}\left(C_{i j}\right)\right) \cdot\left(1-\mu_{B}\left(C_{i j}\right)-\nu_{B}\left(C_{i j}\right)\right)
\end{array}\right\}}{\left\{\left(1-\nu_{A_{i}}\left(C_{i j}\right)\right)^{2}+\left(1-\mu_{A_{i}}\left(C_{i j}\right)\right)^{2}+2\left(1-\mu_{A_{i}}\left(C_{i j}\right)-\nu_{A_{i}}\left(C_{i j}\right)\right)^{2}\right\} .} \\
& \sqrt{ }\left\{\left(1-\nu_{B}\left(C_{i j}\right)\right)^{2}+\left(1-\mu_{B}\left(C_{i j}\right)\right)^{2}+2\left(1-\mu_{B}\left(C_{i j}\right)-\nu_{B}\left(C_{i j}\right)\right)^{2}\right\}
\end{aligned}
$$

or $W I C_{I F S}$ measure:

$$
W I C_{I F S}\left(A_{i}, B\right)=\sum_{j=1}^{n} \omega_{j} \frac{\left\{\begin{array}{l}
\left(1-\nu_{A_{i}}\left(C_{i j}\right)\right) \cdot\left(1-\nu_{B}\left(C_{i j}\right)\right)+\left(1-\mu_{A_{i}}\left(C_{i j}\right)\right) \cdot\left(1-\mu_{B}\left(C_{i j}\right)\right) \\
+2\left(1-\mu_{A_{i}}\left(C_{i j}\right)-\nu_{A_{i}}\left(C_{i j}\right)\right) \cdot\left(1-\mu_{B}\left(C_{i j}\right)-\nu_{B}\left(C_{i j}\right)\right)
\end{array}\right\}}{\sqrt{\frac{\left\{\left(1-\nu_{A_{i}}\left(C_{i j}\right)\right)^{2}+\left(1-\mu_{A_{i}}\left(C_{i j}\right)\right)^{2}+2\left(1-\mu_{A_{i}}\left(C_{i j}\right)-\nu_{A_{i}}\left(C_{i j}\right)\right)^{2}\right\}}{\left\{\left(1-\nu_{B}\left(C_{i j}\right)\right)^{2}+\left(1-\mu_{B}\left(C_{i j}\right)\right)^{2}+2\left(1-\mu_{B}\left(C_{i j}\right)-\nu_{B}\left(C_{i j}\right)\right)^{2}\right\}}}}
$$

to compute the measure values between the alternatives $A_{i}(i=1,2, \ldots, m)$ and the ideal alternative $B$.

(Step 3:) Rank all the alternatives based on index as obtained from $k=\arg \max \left\{I C_{I F S}\right\}$ or $k=\arg \max \left\{W I C_{I F S}\right\}$. More the measure index, the better the alternative $A_{i}(i=1,2, \ldots, m)$.

4.2. Illustrative Example. The above mentioned approach has been demonstrated with a numerical example on pattern recognition; medical diagnosis and an investing a money in a market. The details illustration have been described as follows.

4.2.1. Pattern recognition. Consider a universe $X=\left\{x_{1}, x_{2}, x_{3}\right\}$ and three known pattern $C_{1}, C_{2}$ and $C_{3}$ in the form of IFSs given as

$$
\begin{aligned}
& C_{1}=\left\{\left(x_{1}, 1.0,0.0\right),\left(x_{2}, 0.8,0.0\right),\left(x_{3}, 0.7,0.1\right)\right\} \\
& C_{2}=\left\{\left(x_{1}, 0.8,0.1\right),\left(x_{2}, 1.0,0.0\right),\left(x_{3}, 0.9,0.0\right)\right\} \\
& C_{3}=\left\{\left(x_{1}, 0.6,0.2\right),\left(x_{2}, 0.8,0.0\right),\left(x_{3}, 1.0,0.0\right)\right\}
\end{aligned}
$$

Consider an unknown IFS pattern $P=\left\{\left(x_{1}, 0.5,0.3\right),\left(x_{2}, 0.6,0.2\right),\left(x_{3}, 0.8,0.1\right)\right\}$ which will be recognized. Then the target is to classify the pattern $P$ in one of the classes of $C_{1}, C_{2}$ and $C_{3}$. For it, an improved cosine similarity measure (3.4) has been utilized to compute the measurement values from $P$ to $C_{k}(k=1,2,3)$ and their corresponding results for each pattern is given as follows.

$$
I C_{I F S}\left(C_{1}, P\right)=0.9085 ; \quad I C_{I F S}\left(C_{2}, P\right)=0.9191 ; \quad I C_{I F S}\left(C_{3}, P\right)=0.9736
$$

Thus, based on the recognition principal, we conclude that pattern $P$ should be classified with $C_{3}$.

On the other hand, if we apply the similarity measure, as proposed by Ye [29], to classify the pattern $P$ then their measurement values are computed as $C_{I F S}\left(C_{1}, P\right)=$ $0.9353, C_{I F S}\left(C_{2}, P\right)=0.9519$ and $C_{I F S}\left(C_{3}, P\right)=0.9724$. On the other hand, by using the similarity measure $S_{D C}$ as proposed by Dengfeng and Chuntian [6] to the considered data then their corresponding measurement values are obtained as $S_{D C}\left(C_{1}, P\right)=0.74$, $S_{D C}\left(C_{2}, P\right)=0.78, S_{D C}\left(C_{3}, P\right)=0.84$ while by using the similarity measure $T$ as proposed by Liu [22] measure, then their corresponding results are $T\left(C_{1}, P\right)=0.72$, $T\left(C_{2}, P\right)=0.74, T\left(C_{3}, P\right)=0.84$. Hence, from all these analysis, it has been concluded 
that the pattern $P$ is recognized with the $C_{3}$ and which coincides with the proposed measure result.

If we assign that weight vector of the elements $x_{1}, x_{2}$, and $x_{3}$ be $0.5,0.3$, and 0.2 , then by using Dengfeng and Chuntian [6] measure (corresponding to $p=2$ ), we get $S_{D C}\left(C_{1}, P\right)=0.696, S_{D C}\left(C_{2}, P\right)=0.779$ and $S_{D C}\left(C_{3}, P\right)=0.853$ while by Ye [29] we get $W_{I F S}\left(C_{1}, P\right)=0.9133, W_{I F S}\left(C_{2}, P\right)=0.9404$ and $W_{I F S}\left(C_{3}, P\right)=0.9712$. On the other hand, if we utilize the proposed weighted cosine similarity measure defined in Eq. (3.7), then their corresponding measurement values are

$$
W I C_{I F S}\left(C_{1}, P\right)=0.8737 ; W I C_{I F S}\left(C_{2}, P\right)=0.9103 ; W I C_{I F S}\left(C_{3}, P\right)=0.9775
$$

Hence, pattern $P$ should be classified with $C_{3}$.

Finally, the measurement values of the various existing similarity measures have been computed for the considered data and their corresponding analysis and results are summarized in Table 2. From this table, we conclude that pattern $P$ belongs to the pattern $C_{3}$ too and the result coincides with the existing similarity measure results and validate the approach.

Table 2. Similarity measure comparison for Pattern recognition

\begin{tabular}{c|ccc}
\hline & $\left(P, Q_{1}\right)$ & $\left(P, Q_{2}\right)$ & $\left(P, Q_{3}\right)$ \\
\hline$S_{C}[4,5]$ & 0.7500 & 0.7667 & 0.9000 \\
$S_{H}[20]$ & 0.7500 & 0.7667 & 0.9000 \\
$S_{L}[7]$ & 0.7500 & 0.7667 & 0.9000 \\
$S_{O}[7]$ & 0.7142 & 0.7551 & 0.8845 \\
$S_{D C}[6]$ & 0.7500 & 0.7667 & 0.9000 \\
$S_{H B}[23]$ & 0.7500 & 0.7667 & 0.9000 \\
$S_{e}^{p}[31]$ & 0.7500 & 0.7667 & 0.9000 \\
$S_{s}^{p}[31]$ & 0.7500 & 0.7667 & 0.9000 \\
$S_{H Y}^{1}[21]$ & 0.7000 & 0.7333 & 0.8667 \\
$S_{H Y}^{2}[21]$ & 0.5900 & 0.6297 & 0.8025 \\
$S_{H Y}^{3}[21]$ & 0.5385 & 0.5789 & 0.7647 \\
$C_{I F S}[29]$ & 0.9353 & 0.9519 & 0.9724 \\
$I C_{I F S}$ (proposed) & 0.9085 & 0.9191 & 0.9736 \\
\hline
\end{tabular}

4.2.2. Medical diagnosis. Consider a set of diagnoses $Q=\left\{Q_{1}\right.$ (Viral fever), $Q_{2}$ (Malaria), $Q_{3}$ (Typhoid), $Q_{4}$ (Stomach Problem), $Q_{5}$ (Chest problem) $\}$ and a set of symptoms $S=$ $\left\{s_{1}\right.$ (Temperature), $s_{2}$ (HeadAche), $s_{3}$ (Stomach Pain), $s_{4}$ (Cough), $s_{5}$ (Chest pain) $\}$ which are represented in the form of IFSs as below.

$$
\begin{aligned}
& Q_{1}=\left\{\left(s_{1}, 0.4,0.0\right),\left(s_{2}, 0.3,0.5\right),\left(s_{3}, 0.1,0.7\right),\left(s_{4}, 0.4,0.3\right),\left(s_{5}, 0.1,0.7\right)\right\} \\
& Q_{2}=\left\{\left(s_{1}, 0.7,0.0\right),\left(s_{2}, 0.2,0.6\right),\left(s_{3}, 0.0,0.9\right),\left(s_{4}, 0.7,0.0\right),\left(s_{5}, 0.1,0.8\right)\right\} \\
& Q_{3}=\left\{\left(s_{1}, 0.3,0.3\right),\left(s_{2}, 0.6,0.1\right),\left(s_{3}, 0.2,0.7\right),\left(s_{4}, 0.2,0.6\right),\left(s_{5}, 0.1,0.9\right)\right\} \\
& Q_{4}=\left\{\left(s_{1}, 0.1,0.7\right),\left(s_{2}, 0.2,0.4\right),\left(s_{3}, 0.8,0.0\right),\left(s_{4}, 0.2,0.7\right),\left(s_{5}, 0.2,0.7\right)\right\} \\
& Q_{5}=\left\{\left(s_{1}, 0.1,0.8\right),\left(s_{2}, 0.0,0.8\right),\left(s_{3}, 0.2,0.8\right),\left(s_{4}, 0.2,0.8 .,\left(s_{5}, 0.8,0.1\right)\right\}\right.
\end{aligned}
$$

Suppose a patient $P$ has been evaluated by an expert in order to find which diseases are the most affected by the person. For it, they have treated this patient as a reference set and gave their preferences with respect to all the symptoms in terms of IFNs and represented by the following set:

$$
P=\left\{\left(s_{1}, 0.8,0.1\right),\left(s_{2}, 0.6,0.1\right),\left(s_{3}, 0.2,0.8\right),\left(s_{4}, 0.6,0.1\right),\left(s_{5}, 0.1,0.6\right)\right\}
$$


Now, the target is to classify the patient $P$ in one of the $Q_{1}, Q_{2}, Q_{3}, Q_{4}, Q_{5}$. For this, a developed cosine similarity measure $I C_{I F S}$ as given in Eq. (3.4) has been utilized and their following indices have been computed corresponding to it.

$$
\begin{array}{rr}
I C_{I F S}\left(P, Q_{1}\right)=0.9264 ; \quad I C_{I F S}\left(P, Q_{2}\right)=0.9376 ; \quad I C_{I F S}\left(P, Q_{3}\right)=0.8900 ; \\
I C_{I F S}\left(P, Q_{4}\right)=0.7010 ; \quad I C_{I F S}\left(P, Q_{5}\right)=0.6438
\end{array}
$$

Thus, ranking order of diseases is $Q_{2} \succ Q_{1} \succ Q_{3} \succ Q_{4} \succ Q_{5}$ and hence the patient $P$ suffer from $Q_{2}$ (Malaria) disease.

On the other hand, if we compute the similarity index by using existing Ye [29] approach then their corresponding results are $C_{I F S}\left(P, Q_{1}\right)=0.9046, C_{I F S}\left(P, Q_{2}\right)=0.8832$, $C_{I F S}\left(P, Q_{3}\right)=0.8510, C_{I F S}\left(P, Q_{4}\right)=0.5033$ and $C_{I F S}\left(P, Q_{5}\right)=0.4190$. The detailed analysis of the considered problem by using the existing similarity as well as cosine similarity indices are summarized in Table 3. From these results, we have concluded that the patient suffer from the diagnosis $Q_{1}$ (Viral fever). Also, it has been seen that the existing approaches does not coincide with the proposed one because the proposed approach have also considered the pairs of indeterminacy between the membership functions, while the existing approaches have ignored it.

Table 3. Similarity measure comparison for Medical diagnosis

\begin{tabular}{c|ccccc}
\hline & $\left(P, Q_{1}\right)$ & $\left(P, Q_{2}\right)$ & $\left(P, Q_{3}\right)$ & $\left(P, Q_{4}\right)$ & $\left(P, Q_{5}\right)$ \\
\hline$S_{C}[4,5]$ & 0.6300 & 0.6400 & 0.8200 & 0.6400 & 0.8200 \\
$S_{H}[20]$ & 0.5900 & 0.6400 & 0.8000 & 0.6400 & 0.8000 \\
$S_{L}[7]$ & 0.6100 & 0.6400 & 0.8100 & 0.6400 & 0.8100 \\
$S_{O}[7]$ & 0.5194 & 0.5805 & 0.6714 & 0.5901 & 0.7000 \\
$S_{D C}[6]$ & 0.6300 & 0.6400 & 0.8200 & 0.6400 & 0.8200 \\
$S_{H B}[23]$ & 0.5900 & 0.6400 & 0.8000 & 0.6400 & 0.8000 \\
$S_{e}^{p}[31]$ & 0.5900 & 0.6400 & 0.8000 & 0.6400 & 0.8000 \\
$S_{s}^{p}[31]$ & 0.6200 & 0.6400 & 0.8100 & 0.6400 & 0.8100 \\
$S_{H Y}^{1}[21]$ & 0.5200 & 0.6000 & 0.7600 & 0.5800 & 0.7200 \\
$S_{H Y}^{2}[21]$ & 0.3969 & 0.4785 & 0.6625 & 0.4575 & 0.6137 \\
$S_{H Y}^{3}[21]$ & 0.3514 & 0.4286 & 0.6129 & 0.4085 & 0.5625 \\
$C_{I F S}[29]$ & 0.9046 & 0.8832 & 0.8510 & 0.5033 & 0.4190 \\
$I C_{I F S}$ (proposed) & 0.9264 & 0.9376 & 0.8900 & 0.7010 & 0.6438 \\
\hline
\end{tabular}

4.2.3. Invest a money. Consider a person which have some sort of money and they want to invest in some multi-national company. After carefully analyzing the market, they have analyzed the following four possible alternative namely, car, food, computer and arm companies denoted by $A_{i}, i=1,2,3,4$ respectively. The decision maker take a decision by keeping three criteria on his mind namely risk, growth and environment impact factor denoted by $C_{j}, j=1,2,3$ respectively and giving their weights according to it as $\omega=(0.35,0.25,0.40)^{T}$. The information related to it has been summarized in the form of decision matrix as below.

$$
D=\left(\begin{array}{ccc}
\langle 0.4,0.3\rangle & \langle 0.4,0.2\rangle & \langle 0.1,0.5\rangle \\
\langle 0.6,0.2\rangle & \langle 0.6,0.2\rangle & \langle 0.4,0.1\rangle \\
\langle 0.3,0.3\rangle & \langle 0.5,0.3\rangle & \langle 0.5,0.1\rangle \\
\langle 0.7,0.1\rangle & \langle 0.6,0.1\rangle & \langle 0.3,0.1\rangle
\end{array}\right)
$$

In order to access the best alternative in the decision set, a concept of an ideal point has been used. Although the ideal alternative does not exist in real world, it does provide a useful theoretical construct against which to evaluate alternatives. Hence, we define 
the fixed ideal alternative $A^{*}=\langle 1,0\rangle$ as the IFS. Now, utilizing the proposed weighted cosine similarity measure as defined in Eq. (3.7) to compute the measurement values of each alternative from its ideal alternative and hence get

$$
\begin{array}{ll}
W I C_{I F S}\left(A_{1}, A^{*}\right)=0.5857 \quad ; \quad W I C_{I F S}\left(A_{2}, A^{*}\right)=0.7903 \\
W I C_{I F S}\left(A_{3}, A^{*}\right)=0.7146 \quad ; \quad W I C_{I F S}\left(A_{4}, A^{*}\right)=0.7813
\end{array}
$$

Thus, the ranking order of the alternatives $A_{i}(i=1,2,3,4)$ is $A_{2} \succ A_{4} \succ A_{3} \succ A_{1}$ where $\succ$ means "preferred to".

On the other hand, if we apply Ye [29] approach to compute the cosine similarity measure index corresponding to each alternative, then their corresponding measurement values are $W C_{I F S}\left(A_{1}, A^{*}\right)=0.5821, W C_{I F S}\left(A_{2}, A^{*}\right)=0.9573, W C_{I F S}\left(A_{3}, A^{*}\right)=0.8541$ and $W C_{I F S}\left(A_{4}, A^{*}\right)=0.9726$. Finally, in order to analyze the effect of the weight vector on the selection of the best alternative(s), we have conducted the analysis and their correspondingly results are summarized in Table 4 . From this table, it has been seen that the best alternative is $A_{2}$ i.e., food company while by Ye [29] result, the best alternative is $A_{4}$. But it has been observed as above that Ye [29] does not consider the pair of the interaction between the pairs of the membership functions and hence their corresponding results does not give the correct decision to the system analysts.

Table 4. Effect of weight vector on cosine similarity measure index

\begin{tabular}{c|c|c}
\hline$\omega$ & Ye [29] result & Proposed results \\
\hline$(1 / 3,1 / 3,1 / 3)$ & $(0.6302,0.9558,0.8484,0.9750)$ & $(0.6039,0.8007,0.7177,0.7939)$ \\
$(0.25,0.25,0.50)$ & $(0.5217,0.9594,0.8814,0.9684)$ & $(0.5593,0.7746,0.7298,0.7537)$ \\
$(0.35,0.25,0.40)$ & $(0.5821,0.9573,0.8541,0.9726)$ & $(0.5857,0.7903,0.7146,0.7813)$ \\
$(0.40,0.25,0.35)$ & $(0.6122,0.9562,0.8404,0.9746)$ & $(0.5989,0.7981,0.7070,0.7951)$ \\
$(0.50,0.30,0.20)$ & $(0.7076,0.9530,0.8069,0.9806)$ & $(0.6389,0.8215,0.6921,0.8330)$ \\
$(0.25,0.40,0.35)$ & $(0.6264,0.9562,0.8630,0.9741)$ & $(0.5999,0.7981,0.7308,0.7846)$ \\
\hline
\end{tabular}

\section{Conclusion}

In this paper, we have presented newly improved cosine and weighted cosine similarity measures based decision-making method between the intuitionistic fuzzy sets. For it, firstly some drawbacks of the existing cosine similarity measures have been pointed out and validated with a numerical example. These shortcomings have been resolved by defining new operational laws between the two IFSs by considering the pairs of the interaction between their membership functions. Based on these new operations, informational energies between the sets of IFNs as well as cosine similarity measures index have been proposed under the intuitionistic fuzzy environment. An illustrated example has been given which demonstrate the proposed similarity measure can easily handle the situation where the existing measures fail. Also, the developed measures have been tested on some pattern recognition, medical and MCDM problems which show the strength of the approach. By comparison with the existing paper's results with the proposed results, it has been found that the proposed results are more stable and practical and coincides with the conclusion of the existing measures. In our further research, we will focus on adopting this approach to some more complicated applications from the fields of cluster analysis, uncertain programming, and mathematical programming. 


\section{References}

[1] Atanassov, K. T., Intuitionistic fuzzy sets, Fuzzy Sets Syst., 20, 87 - 96, 1986.

[2] Atanassov, K., Gargov, G., Interval-valued intuitionistic fuzzy sets, Fuzzy Sets Syst., 31, $343-349,1989$.

[3] Bustince, H., Burillo, P., Correlation of interval-valued intuitionistic fuzzy sets,Fuzzy Sets Syst., 74, $237-244,1995$.

[4] Chen, S. M., Measures of similarity between vague sets, Fuzzy Sets Syst., 74 (2), $217-223$, 1995.

[5] Chen, S. M., Similarity measures between vague sets and between elements, IEEE Transact. Systems Man Cybernet. 27 (1), 153 - 158, 1997.

[6] Dengfeng, L., Chuntian, C., New similarity measure of intuitionistic fuzzy sets and application to pattern recognitions, Pattern Recognition Lett. 23, 221 - 225, 2002.

[7] Fan, L., Zhangyan, X., Similarity measures between vague sets., J. Software 12 (6), 922 927, 2001.

[8] Garg, H., Generalized intuitionistic fuzzy interactive geometric interaction operators using Einstein t-norm and t-conorm and their application to decision making, Comput. Industrial Eng. 101, 53-69, 2016.

[9] Garg, H., Generalized intuitionistic fuzzy multiplicative interactive geometric operators and their application to multiple criteria decision making, Int. J. Mach. Learn. Cybernet. 7 (6), $1075-1092,2016$.

[10] Garg, H., A new generalized improved score function of interval-valued intuitionistic fuzzy sets and applications in expert systems, Appl. Soft Comput. 38, 988 - 999, 2016.

[11] Garg, H., Distance and similarity measure for intuitionistic multiplicative preference relation and its application, Int. J. Uncertain. Quantif. 7 (2), 117 - 133, 2017.

[12] Garg, H., Novel intuitionistic fuzzy decision making method based on an improved operation laws and its application, Eng. Appli. Artif. Intell. 60, $164-174,2017$.

[13] Garg, H., Agarwal, N., Choubey, A., Entropy based multi-criteria decision making method under fuzzy environment and unknown attribute weights, Global J. Technol. Optim. 6 (3), $13-20,2015$.

[14] Garg, H., Agarwal, N., Tripathi, A., Generalized intuitionistic fuzzy entropy measure of order $\alpha$ and degree $\beta$ and its applications to multi-criteria decision making problem, Int. J. Fuzzy Syst. Appl. 6 (1), 86 - 107, 2017.

[15] Garg, H., Arora, R., Distance and similarity measures for dual hesistant fuzzy soft sets and their applications in multi criteria decision-making problem, Int. J. Uncertain. Quantif., 7 (3), 229-248, 2017.

[16] Garg, H., Rani, M., Sharma, S. P., Vishwakarma, Y., Intuitionistic fuzzy optimization technique for solving multi-objective reliability optimization problems in interval environment, Expert Systems with Applications 41, 3157 - 3167, 2014.

[17] Gerstenkorn, T., Manko, J., Correlation of intuitionistic fuzzy sets, Fuzzy Sets Syst., 44, 39 $-43,1991$.

[18] He, Y., Chen, H., Zhau, L., Liu, J., Tao, Z., Intuitionistic fuzzy geometric interaction averaging operators and their application to multi-criteria decision making, Information Sci., 259, 142 - 159, 2014.

[19] He, Y., Chen, H, Zhou, L., Han, B., Zhao, Q., Liu, J., Generalized intuitionistic fuzzy geometric interaction operators and their application to decision making, Expert Syst. Appl., 41 (0), $2484-2495,2014$.

[20] Hong, D. H., Kim, C., A note on similarity measures between vague sets and between elements, Information Sci., 115, 83 - 96, 1999.

[21] Hung, W. L., Yang, M. S., Similarity measures of intuitionistic fuzzy sets based on hausdorff distance, Pattern Recognition Lett. 25, 1603 - 1611, 2004.

[22] Liu, H. W., New similarity measures between intuitionistic fuzzy sets and between elements, Math. Comput. Modelling 42, $61-70,2005$.

[23] Mitchell, H. B., On the dengfeng chuntian similarity measure and its application to pattern recognition, Pattern Recognition Lett. 24, 3101 - 3104, 2003. 
[24] Singh, S., Garg, H., Distance measures between type-2 intuitionistic fuzzy sets and their application to multicriteria decision-making process, Applied Intelligence 46 (4), 788 - 799, 2017.

[25] Szmidt, E., Kacprzyk, J., A similarity measure for intuitionistic fuzzy sets and its application in supporting medical diagnostic reasoning, Lecture Notes in Computer Science 3070, 388 $393,2004$.

[26] Xu, Z. S., On correlation measures of intuitionistic fuzzy sets, Lecture Notes in Computer Science 4224, $16-24,2006$.

[27] Xu, Z. S., Intuitionistic fuzzy aggregation operators, IEEE Trans. Fuzzy Systems 15, 1179 $-1187,2007$.

[28] Xu, Z. S., Intuitionistic preference relations and their application in group decision making, Information Sci., 177, 2363 - 2379, 2007.

[29] Ye, J., Cosine similarity measures for intuitionistic fuzzy sets and their applications, Math. Comput. Modelling 53, 91 - 97, 2011.

[30] Zadeh, L. A., Fuzzy sets, Information and Control 8, 338-353, 1965.

[31] Zhizhen, L., Pengfei, S., Similarity measures on intuitionistic fuzzy sets, Pattern Recognition Lett. 24, $2687-2693,2003$. 